\title{
Lack of prognostic relevance of Her-2/neu, topoisomerase II $\alpha$ and EGFR in advanced ovarian carcinoma
}

\author{
VERENA ENGELSTAEDTER ${ }^{1 *}$, JUDITH BODA $^{2 *}$, CHRISTINE VÖLKLEIN $^{2}$, JUTTA ENGEL $^{3}$, \\ UDO JESCHKE $^{1}$, THOMAS KIRCHNER ${ }^{2}$ and DORIS MAYR ${ }^{2}$ \\ ${ }^{1}$ Department of Obstetrics and Gynecology, Campus Innenstadt; ${ }^{2}$ Institute of Pathology, Ludwig-Maximilians University, \\ D-80337 Munich; ${ }^{3}$ Munich Cancer Registry, Ludwig-Maximilians University, D-81377 Munich, Germany
}

Received October 25, 2011; Accepted January 11, 2012

DOI: $10.3892 /$ etm.2012.481

\begin{abstract}
Patients with advanced ovarian cancer (FIGO stage III) have a poor clinical prognosis. However, these patients show distinct differences in their survival time, possibly due to differing responses to chemotherapy and differing tumor biology. In contrast to histological subtype, grading and staging, which are known to affect a patient's prognosis, the impact of the human epidermal growth factor receptor 2 (Her-2/neu), topoisomerase II $\alpha$ and epidermal growth factor receptor (EGFR) on survival remain inconclusive. Therefore, the aim of this study was to assess their impact on survival in a group of advanced ovarian cancer patients. Tissue microarrays were constructed from specimens of 243 patients. Gene copy and chromosome numbers were evaluated by fluorescence in situ hybridization (FISH) and protein expression by immunohistochemistry (IHC). Scoring for the latter was calculated by considering the percentage of positive tumor cells and the relative staining intensity. FISH results were evaluated by previously published recommendations and correlated with overall survival. Using IHC, $1.6 \%$ of the cases that were tested for Her-2/neu and topoisomerase II $\alpha$ were strongly positive, and $12.3 \%$ were positive for EGFR. Using FISH, 4.4\% amplifications and $2.1 \%$ polysomies for Her-2/neu were identified; topoisomerase II $\alpha$ showed $2.2 \%$ amplifications, $0.4 \%$ deletions and $3.5 \%$ polysomies. We observed $10.8 \%$ high polysomies, but no amplification for EGFR. None of the results obtained by IHC or FISH correlated with overall survival. In general, Her-2/neu, topoisomerase II $\alpha$ and EGFR may be prognostic factors in ovarian carcinomas. However, within this group
\end{abstract}

Correspondence to: Dr Verena Engelstaedter, Department of Obstetrics and Gynecology, Ludwig-Maximilians University, Maistrasse 11, D-80337 Munich, Germany

E-mail: verena.engelstaedter@med.uni-muenchen.de

${ }^{*}$ Contributed equally

Key words: advanced ovarian cancer, Her-2/neu, topoisomerase II $\alpha$, epidermal growth factor receptor, co-amplification, prognosis, fluorescence in situ hybridization of FIGO stage III patients, differences in gene aberration or protein expression were not able to predict differences in survival.

\section{Introduction}

Ovarian cancer is the most deadly gynecological malignancy with approximately 9700 newly diagnosed cases and 5600 mortalities in Germany each year $(1,2)$. Regarding mortality, it ranks fifth after breast, intestinal, lung and uterine cancer $(3,4)$. The median age at time of diagnosis at all stages is 68 years.

Early symptoms of ovarian cancer are rare and non-specific as they often mimic those of other more common conditions, such as menopausal or digestive disorders (5). Thus, approximately $75 \%$ of all patients with ovarian cancer are diagnosed at FIGO stage III, when the disease has spread to the upper abdomen or lymph nodes and is often only suitable for palliative therapy (6). The chance for cure in this advanced stage is $10 \%$ (4) and to date the 5-year survival rate is no more than $47 \%(1,2)$. Platinum-based combination chemotherapy is the standard treatment for patients with ovarian cancer, but the low survival rates demand a more effective or more specific treatment. Within the last few years, several chemotherapy regimens have been tested showing little or no improvement in survival. Focusing on FIGO stage III patients, it is interesting that in spite of identical tumor stage and therapy, the survival times vary considerably from patient to patient $(7,8)$. This raises the question of whether further tumor biological factors affect the course of disease and the effectiveness of therapies. While the investigation of biological factors such as the human epidermal growth factor receptor 2 (Her-2/neu) are carried out as standard procedures in patients with breast cancer, these tests are rarely performed in patients with ovarian cancer (9). New therapeutic strategies focus on the combination of molecular-targeted therapies with cytotoxic agents, which could lead to an improvement in treatment response and patient outcome (10). The oncogenes epidermal growth factor receptor (EGFR) and Her-2/neu have been studied as prognostics factors in ovarian cancer and these markers hold considerable promise as therapeutic agents. However, their prognostic value in terms of survival, particularly in advanced stage patients, remains unknown (11). Topoisomerase II $\alpha$ 
(Top IIa) is a nuclear enzyme that is essential for DNA metabolism, including DNA replication, transcription, recombination and chromosome condensation (12). As with Her-2/neu and EGFR, Top IIa is a molecular target for antineoplastic agents, but few studies have examined Top IIa expression in ovarian cancer and its prognostic value in terms of overall survival.

The aim of this study was to assess the impact of Her-2/neu, Top IIa and EGFR on the overall survival of advanced stage ovarian cancer patients evaluated by immunohistochemistry (IHC) and fluorescence in situ hybridization (FISH).

\section{Materials and methods}

In the present study, we used paraffin-embedded tissue from 243 unselected patients at a mean age of 57.9 years (range, 26.7-89.1), including 181 serous, 19 mucinous and 17 endometrioid tumors, and 18 undifferentiated carcinomas. Eight cases had multiple histological descriptions and were classified as mixed type carcinomas (the second and third cell type account for at least $10 \%$ of the tumor tissue), mostly of serous and mucinous subtypes. Only very few cases of clear cell carcinomas occurred at our institution and were excluded from the analysis for statistical reasons. The tissue was collected during primary tumor debulking surgery.

Seven (2.9\%) patients were grade I, 88 (36.2\%) were grade II, and $148(60.9 \%)$ were grade III or IV. As there is no internationally accepted grading system for ovarian cancer, we used a three-stage system as recommended by the World Health Organization (13): For serous and mucinous subtypes the growth pattern, cell nucleus pleomorphy and mitosis rate were considered. Endometroid subtypes were graded as grade 1 for glandular histology with $<5 \%$ solid fraction, as grade 2 for glandular histology and a solid fraction of $5-50 \%$, and as grade 3 for glandular histology and a solid fraction of $50 \%$ and above. All cases were classified as FIGO stage III.

Patients were treated at the Department of Obstetrics and Gynecology, Ludwig-Maximilians University of Munich between 1989 and 2002. All cases were primarily diagnosed as ovarian cancers and none of the patients had received chemotherapy prior to surgery. Patients had given written informed consent for their participation in this study. Complete followup data (mean time, 92 months) were available in 190 cases $(78.2 \%)$.

IHC. For immunohistochemical analysis, paraffin-embedded specimens were cut at $<2 \mu \mathrm{m}$, using conventional histological techniques and transferred to slides (SuperFrost Plus, Menzel, Germany).

The immunohistochemical staining for Her-2/neu was performed automatically with Ventanas Benchmark ${ }^{\circledR}$ XT, using the Her-2/neuTest 4B5. Taking into consideration the new recommendations of the American Society of Clinical Oncology/College of American Pathologists, the Dako scoring system was used for interpretation (14): Score 0, no staining or membrane staining in $<10 \%$ of the tumor cells; score $1+$, a faint or barely perceptible incomplete membrane staining in $>10 \%$ of the tumor cells; score $2+$, a weak to moderate staining of the entire membrane in $>10 \%$ of the tumor cells; and score $3+$, a strong staining of the entire membrane in $>30 \%$ of the tumor cells. Strong staining only in areas of tissue borders was ignored. Prior to this, positive and negative control slides had been examined.

The immunohistochemical staining for Top IIa was performed with the primary antibody, NCL-TOPOIIA (Novocastra, Newcastle upon Tyne, UK), at a dilution of 1:20. Detection was accomplished by a system consisting of a secondary biotin-labeled antibody and streptavidin (LSAB2 System-HRP; Dako, Carpinteria, CA, USA). Staining and counter-staining were run with the chromogen AEC (Zytomed Systems, Berlin, Germany) and haematoxylin (Vector Labs., Burlingame, CA, USA).

To date no consistent scoring system has been established. We used the scoring system described in the study by Remmele and Stegner (15) for interpretation of the Top IIa analysis. Staining intensity, as well as the percentage of positive tumor cells, was considered. For calculating the Remmele score, the staining intensity and percentage of positive tumor cells are multiplied and result in a value between 0 and 12 . According to the score, the results were divided into score 0 to 2 , low expression; score 3 to 6 , mild expression; and score 7 to 12 , strong expression [as described previously (16)].

The immunohistochemical staining for the EGFR receptor was performed automatically with Ventanas Benchmark XT, using the EGFR test Confirm EGFR 3C6.

For interpretation of the EGFR test, the Dako interpretation guide was used. EGFR-negative staining was recorded in absence of membrane staining above the background level in all tumor cells. EGFR-positive staining was recorded when there was any IHC staining of tumor cell membranes above the background level; whether this was complete or incomplete circumferential staining.

FISH. For FISH analysis paraffin-embedded specimens were cut at $2 \mu \mathrm{m}$, using conventional histological techniques, and transferred to slides (SuperFrost Plus). Specimens were deparaffinized and rehydrated in two changes of xylene for $30 \mathrm{~min}$ each, two 10-min washes in absolute ethanol and dried at room temperature. A total of 200-300 $\mu \mathrm{l}$ of $1 \mathrm{M}$ sodium thiocyanate was applied to each section. They were then placed in a thermostat bath for $30 \mathrm{~min}$ at $80^{\circ} \mathrm{C}$ and rinsed in deionized water. Following incubation in protease solution $(80 \mu \mathrm{l}$ pepsin stock + $920 \mu$ l deionized water, $\mathrm{pH} 2.0$ ) for $30 \mathrm{~min}$ at $37^{\circ} \mathrm{C}$, the enzymatic reaction was stopped by rinsing the slides with deionized water. They were dried at room temperature. Subsequently, specimens were put on a heater for $20 \mathrm{~min}$ at $80^{\circ} \mathrm{C}$ in order to denature the DNA. Next, $18 \mu \mathrm{l}$ of DNA probe mixture were applied (for Her-2/neu: PathVysion Her-2 DNA Probe Kit, Abbott Molecular Inc., Des Plaines, IL, USA; for Top IIa: LSI TOP 2A Spectrum Orange/CEP 17 Spectrum Green, Abbott Molecular Inc.; and for EGFR: SPEC EGFR/CEN 7 Dual Color Probe, Zytomed Systems, Berlin, Germany). Coverslips were sealed with rubber cement and put on the heater again for $10 \mathrm{~min}$, followed by incubation overnight $(14-18 \mathrm{~h})$ in a thermostat bath $\left(37^{\circ} \mathrm{C}\right)$. Afterwards, slides were washed three times, for $10 \mathrm{~min}$ each, at $42^{\circ} \mathrm{C}$ in $0.1 \mathrm{X} \mathrm{SSC}$-buffer to remove any unspecific bound probes and for another $5 \mathrm{~min}$ at $42^{\circ} \mathrm{C}$ in BT-buffer. Slides were air-dried in the dark and $25 \mu \mathrm{l}$ of 4,6-diamidino-2-phenylindole2-hydrochloride (DAPI) were applied.

Evaluation of FISH analysis was carried out with fluorescence microscopy using the DAPI filter for search and selection 
of tumor nuclei; a FITC filter was used for green signals and TRITC filter for red signals. The filters could be switched by a remote control (Mac 5000; LEP, Hawthorne, NY, USA). A total of 60 cells per case were scored for red and green signals. Overlapping cells, cells without clear margins, and/or with strong background fluorescence were excluded.

For Her-2/neu the interpretation was carried out as described in the study by Pauletti et al (17) in consideration of the new recommendations of the American Society of Clinical Oncology/College of American Pathologists (14): A gene-centromere-ratio (ISH ratio) of $<1.8$ was considered negative for Her-2/neu gene amplification; an ISH ratio of $>2.2$ was considered positive for Her-2/neu gene amplification; and an ISH ratio of 1.8-2.2 (after counting 100 nuclei per sample and after repeating the ISH) was equivocal for Her-2/neu gene amplification.

The interpretation of Top IIa was carried out according to the instructions of the manufacturer (Dako TOP2A FISH pharmDx ${ }^{\mathrm{TM}}$ kit, 3rd edition). A gene/centromere ratio of $<0.8$, Top IIa deletion; a gene/centromere ratio of $\geq 2.0$, Top IIa amplification; a gene/centromere ratio between $0.8-1.9$, no Top IIa amplification. Additionally, the guidelines described by Pauletti et al (17) were used in cases of polysomies and monosomies.

The EGFR probe was evaluated according to the guidelines described by Cappuzzo et al (18). This classification describes six FISH strata with an ascending number of copies of the EGFR gene per cell according to the frequency of tumor cells with a specific number of copies of the EGFR gene and chromosome 7 centromere. The six strata were: Disomy $(\leq 2$ copies in $>90 \%$ of cells); low trisomy ( $\leq 2$ copies in $\geq 40 \%$ of cells, 3 copies in $10-40 \%$ of the cells, and $\geq 4$ copies in $<10 \%$ of cells); high trisomy ( $\leq 2$ copies in $\geq 40 \%$ of cells, 3 copies in $\geq 40 \%$ of cells, and $\geq 4$ copies in $<10 \%$ of cells); low polysomy ( $\geq 4$ copies in $10-40 \%$ of cells); high polysomy ( $\geq 4$ copies in $\geq 40 \%$ of cells); and gene amplification (defined by the presence of tight EGFR gene clusters and a ratio of EGFR gene to chromosome of $\geq 2$ or $\geq 15$ copies of EGFR per cell in $\geq 10 \%$ of the analyzed cells).

Statistical analysis. Documentation and statistical analysis were performed using the SPSS software version 17.0. Co-amplifications and survival time were calculated with cross-classified tables and Kaplan-Meier survival analysis. A p-value $\leq 0.05$ was considered to indicate a statistically significant difference.

\section{Results}

IHC. Her-2/neu was analyzed in 241 of 243 (99.2\%) cases, Top IIa in 227 (93.4\%) cases, and EGFR in 229 (94.2\%) cases. The missing cases were not suitable for evaluation. Immunohistochemical results are shown in Tables I-III.

FISH. FISH analysis for Her-2/neu was carried out in 229 of the 243 (94.2\%) cases. Amplification was observed in ten (4.4\%) cases. Four (1.7\%) cases showed uncertain results. Five (2.2\%) cases presenting polysomy and $26(11.4 \%)$ presenting monosomy were found. The remaining 184 (80.3\%) cases did not show amplification.
Table I. Results of immunohistochemistry analysis in 241 cases of Her-2/neu oncoprotein overexpression.

\begin{tabular}{lcc}
\hline Expression & Number of cases & Percentage \\
\hline Score 0 & 195 & 80.9 \\
Score 1+ & 33 & 13.7 \\
Score 2+ & 9 & 3.7 \\
Score 3+ & 4 & 1.7 \\
All & 241 & 100.0 \\
\hline
\end{tabular}

Her-2/neu, human epidermal growth factor receptor 2 .

Table II. Results of immunohistochemistry analysis in 227 cases of topoisomerase II $\alpha$ overexpression.

\begin{tabular}{lcc}
\hline Expression & Number of cases & Percentage \\
\hline Low & 125 & 55.0 \\
Moderate & 98 & 43.2 \\
Strong & 4 & 1.8 \\
All & 227 & 100.0 \\
\hline
\end{tabular}

Table III. Results of immunohistochemistry analysis in 229 cases of EGFR overexpression.

\begin{tabular}{lcc}
\hline Expression & Number of cases & Percentage \\
\hline Positive & 30 & 13.1 \\
Negative & 199 & 86.9 \\
All & 229 & 100.0 \\
\hline
\end{tabular}

EGFR, epidermal growth factor receptor.

FISH analysis for Top IIa was carried out in in 227 of the $243(93.5 \%)$ cases. Amplification was observed in five $(2.2 \%)$ cases. One $(0.4 \%)$ case showed a deletion. Additionally, eight $(3.5 \%)$ cases presenting polysomy and $33(14.5 \%)$ cases presenting monosomy were found. No amplification was detected in $180(79.3 \%)$ cases.

FISH analysis for EGFR was carried out in 222 of the 243 (91.4\%) cases. In 24 (10.8\%) cases a high polysomy was found. A total of $62(27.9 \%)$ cases had a low polysomy, six (2.7\%) had a high trisomy, 84 (37.8\%) had a low trisomy and 46 (20.7\%) had a disomy. No amplification was observed.

\section{Correlation between IHC and FISH}

Her-2/neu. In the comparison of gene status and protein expression of Her-2/neu, 229 (94.2\%) cases were evaluated. Fourteen (5.8\%) cases had to be excluded (see Table IV). Four $(1.7 \%)$ cases showed amplification using FISH analysis and a strong expression (3+) in the IHC analysis. Three (1.3\%) cases showing amplification showed uncertain results (2+) in the IHC analysis and another three amplifying cases showed a negative Her- 2 test of 0 or $1+$ in the IHC analysis. Four 
Table IV. Comparison of gene status and protein expression of Her-2/neu.

Her-2/neu protein expression score

\begin{tabular}{lccccc}
\cline { 3 - 5 } FISH: Her-2/neu & Number of cases & 0 & $1+$ & $2+$ & $3+$ \\
\hline Normal & $185(80.8 \%)$ & $152(66.4 \%)$ & $29(12.7 \%)$ & $4(1.7 \%)$ & $0(0.0 \%)$ \\
Amplification & $10(4.4 \%)$ & $2(0.9 \%)$ & $1(0.4 \%)$ & $3(1.3 \%)$ & $4(1.7 \%)$ \\
Unclear & $3(1.3 \%)$ & $1(0.4 \%)$ & $1(0.4 \%)$ & $1(0.4 \%)$ & $0(0.0 \%)$ \\
Polysomy & $5(2.2 \%)$ & $3(1.3 \%)$ & $1(0.4 \%)$ & $1(0.4 \%)$ & $0(0.0 \%)$ \\
Monosomy & $26(11.4 \%)$ & $26(11.4 \%)$ & $0(0.0 \%)$ & $0(0.0 \%)$ & $0(0.0 \%)$ \\
All & $229(100 \%)$ & $184(80.3 \%)$ & $32(14.0 \%)$ & $9(3.9 \%)$ & $4(1.7 \%)$
\end{tabular}

Her-2/neu, human epidermal growth factor receptor 2; FISH, fluorescence in situ hybridization.

Table V. Comparison of gene status and protein expression of topoisomerase II $\alpha$.

IHC: Topoisomerase II $\alpha$

\begin{tabular}{lcccc} 
FISH: Topoisomerase II $\alpha$ & Number of cases & Weak expression & Moderate expression & Strong expression \\
\hline Normal & $176(79.3 \%)$ & $100(45.1 \%)$ & $73(32.9 \%)$ & $3(1.4 \%)$ \\
Amplification & $5(2.3 \%)$ & $2(0.9 \%)$ & $3(1.4 \%)$ & $0(0.0 \%)$ \\
Deletion & $1(0.5 \%)$ & $0(0.0 \%)$ & $1(0.5 \%)$ & $0(0.0 \%)$ \\
Polysomy & $8(3.6 \%)$ & $2(1.0 \%)$ & $6(2.8 \%)$ & $0(0.0 \%)$ \\
Monosomy & $32(14.4 \%)$ & $18(8.2 \%)$ & $13(5.9 \%)$ & $1(0.5 \%)$ \\
All & $222(100 \%)$ & $122(55.0 \%)$ & $96(43.2 \%)$ & $4(1.9 \%)$
\end{tabular}

FISH, fluorescence in situ hybridization; ICH, immunohistochemistry.

Table VI. Comparison of gene status and protein expression of ErbB1.

\begin{tabular}{lccc}
\hline & & \multicolumn{2}{c}{ IHC: EGFR } \\
\cline { 3 - 4 } FISH: ErbB1 & Number of cases & Negative & Positive \\
\hline Disomy & $44(20.3 \%)$ & $39(18 \%)$ & $5(2.3 \%)$ \\
Low trisomy & $82(37.8 \%)$ & $73(33.6 \%)$ & $9(4.1 \%)$ \\
High trisomy & $6(2.8 \%)$ & $5(2.3 \%)$ & $1(0.5 \%)$ \\
Low polysomy & $61(28.1 \%)$ & $51(23.5 \%)$ & $10(4.6 \%)$ \\
High polysomy & $24(11.1 \%)$ & $22(10.1 \%)$ & $2(0.9 \%)$ \\
All & $217(100 \%)$ & $190(87.6 \%)$ & $27(12.4 \%)$ \\
\hline
\end{tabular}

FISH, fluorescence in situ hybridization; ICH, immunohistochemistry; EGFR, epidermal growth factor receptor.

uncertain cases in the FISH evaluation coincided with the scores 0 (once), $1+$ (once) and 2+ (twice) in the IHC analysis. Five $(2.2 \%)$ cases of polysomy had negative IHC scores of $0 / 1+(4 / 5,1.7 \%)$ and a moderate IHC score of $2+(1 / 5,0.4 \%)$. All cases of monosomy $(26,11.4 \%)$ showed a negative IHC test. This analysis was significant $(\mathrm{p}<0.0005)$.

Top IIa. A total of 222 (91.4\%) cases were suitable for the comparison of gene status and protein expression of Top IIa,
$21(8.6 \%)$ cases had to be excluded (see Table V). Three $(1.4 \%)$ cases of strong expression revealed a normal gene status. One $(0.5 \%)$ case of strong expression coincided with a monosomy. Thus, no correlation between amplification and strong expression was found. In three (1.4\%) cases of amplification, a moderate level of expression was found. Another two (1.0\%) amplifying cases showed a weak expression in the IHC analysis. Cases of polysomy did not coincide with strong expression either. Six (2.8\%) of those cases had a moderate and two $(1.0 \%)$ had weak expression in the IHC evaluation. The only case $(0.5 \%)$ of deletion also showed moderate expression. No statistical significance was achieved $(\mathrm{p}=0.167)$.

EGFR. A total of 217 (89.3\%) cases were compared, and $26(10.7 \%)$ cases had to be excluded (see Table VI). Only two cases $(0.9 \%)$ showed a correlation between FISH and IHC; a high polysomy in combination with a positive IHC test. A total of 22 of the $24(10.1 \%)$ cases of high polysomy coincided with a negative protein expression. A total of 168 (77.4\%) cases were negative in the FISH analysis as well as in the IHC evaluation. Nine (4.1\%) IHC-positive cases showed low trisomy and ten $(4.6 \%)$ IHC-positive cases had low polysomy. No significant correlation was found $(\mathrm{p}=0.817)$.

\section{Co-amplification}

Her-2/neu and Top IIa. In total, 225 of the 243 (92.6\%) cases were compared in the FISH analysis of Top IIa and Her-2/neu. Eighteen $(7.4 \%)$ cases were not suitable for evaluation; the 
Table VII. Comparison of Her-2/neu and topoisomerase-II $\alpha$ in the FISH analysis.

Topoisomerase II $\alpha$

\begin{tabular}{lcccccc}
\cline { 3 - 6 } Her-2/neu & Number of cases & Normal & Amplification & Deletion & Polysomy & Monosomy \\
\hline Normal & $181(80.4 \%)$ & $169(75.1 \%)$ & $1(0.4 \%)$ & $1(0.4 \%)$ & $0(0.0 \%)$ & $10(4.4 \%)$ \\
Amplification & $10(4.4 \%)$ & $3(1.3 \%)$ & $4(1.8 \%)$ & $0(0.0 \%)$ & $3(1.3 \%)$ & $0(0.0 \%)$ \\
Uncertain & $3(1.3 \%)$ & $2(0.9 \%)$ & $0(0.0 \%)$ & $0(0.0 \%)$ & $1(0.4 \%)$ & $0(0.0 \%)$ \\
Polysomy & $5(2.2 \%)$ & $1(0.4 \%)$ & $0(0.0 \%)$ & $0(0.0 \%)$ & $4(1.8 \%)$ & $0(0.0 \%)$ \\
Monosomy & $26(11.6 \%)$ & $3(1.3 \%)$ & $0(0.0 \%)$ & $0(0.0 \%)$ & $0(0.0 \%)$ & $23(10.2 \%)$ \\
All & $225(100 \%)$ & $178(79.1 \%)$ & $5(2.2 \%)$ & $1(0.4 \%)$ & $8(3.6 \%)$ & $33(14.7 \%)$ \\
\hline
\end{tabular}

Her-2/neu, human epidermal growth factor receptor 2; FISH, fluorescence in situ hybridization.

Table VIII. Comparison of Her-2/neu and ErbB1 in the FISH analysis.

ErbB1

\begin{tabular}{lcccccc}
\cline { 3 - 6 } Her-2/neu & Number of cases & Disomy & Low trisomy & High trisomy & Low polysomy & High polysomy \\
\hline Normal & $179(81.0 \%)$ & $37(16.7 \%)$ & $66(29.9 \%)$ & $5(2.3 \%)$ & $53(24.0 \%)$ & $18(8.1 \%)$ \\
Amplification & $10(4.5 \%)$ & $0(0.0 \%)$ & $4(1.8 \%)$ & $0(0.0 \%)$ & $3(1.4 \%)$ & $3(1.4 \%)$ \\
Uncertain & $3(1.4 \%)$ & $0(0.0 \%)$ & $1(0.5 \%)$ & $0(0.0 \%)$ & $2(0.9 \%)$ & $0(0.0 \%)$ \\
Polysomy & $5(2.3 \%)$ & $0(0.0 \%)$ & $1(0.5 \%)$ & $0(0.0 \%)$ & $2(0.9 \%)$ & $2(0.9 \%)$ \\
Monosomy & $24(10.9 \%)$ & $9(4.1 \%)$ & $12(5.4 \%)$ & $1(0.5 \%)$ & $1(0.5 \%)$ & $1(0.5 \%)$ \\
All & $221(100 \%)$ & $46(20.8 \%)$ & $84(38.0 \%)$ & $6(2.7 \%)$ & $61(27.6 \%)$ & $24(10.9 \%)$
\end{tabular}

Her-2/neu, human epidermal growth factor receptor 2; FISH, fluorescence in situ hybridization.

Table IX. Comparison of topoisomerase II $\alpha$ and ErbB1 in the FISH analysis.

ErbB1

\begin{tabular}{lcccccc} 
Topoisomerase II $\alpha$ & Number of cases & Disomy & Low trisomy & High trisomy & Low polysomy & High polysomy \\
\hline Normal & $175(79.5 \%)$ & $33(15.0 \%)$ & $66(30.0 \%)$ & $5(2.3 \%)$ & $53(24.1 \%)$ & $18(8.2 \%)$ \\
Amplification & $5(2.3 \%)$ & $0(0.0 \%)$ & $3(1.4 \%)$ & $0(0.0 \%)$ & $2(0.9 \%)$ & $0(0.0 \%)$ \\
Deletion & $1(0.5 \%)$ & $0(0.0 \%)$ & $0(0.0 \%)$ & $0(0.0 \%)$ & $0(0.0 \%)$ & $1(0.5 \%)$ \\
Polysomy & $8(3.6 \%)$ & $0(0.0 \%)$ & $0(0.0 \%)$ & $0(0.0 \%)$ & $4(1.8 \%)$ & $4(1.8 \%)$ \\
Monosomy & $31(14.1 \%)$ & $12(5.5 \%)$ & $15(6.8 \%)$ & $1(0.5 \%)$ & $3(1.4 \%)$ & $0(0.0 \%)$ \\
All & $220(100 \%)$ & $45(20.5 \%)$ & $8438.2 \%)$ & $6(2.7 \%)$ & $62(28.2 \%)$ & $23(10.5 \%)$
\end{tabular}

FISH, fluorescence in situ hybridization.

results are demonstrated in Table VII. Four (1.8\%) cases revealed a co-amplification of Her-2/neu and Top IIa. In six (2.6\%) cases, a Her-2/neu amplification was observed, but Top IIa was normal (3 cases) or had a polysomy (3 cases). In four $(1.8 \%)$ cases a polysomy was found in the Her-2/neu and Top IIa analysis. In one (0.4\%) case of the Her-2/neu analysis and in four $(1.8 \%)$ cases of the Top IIa analysis, further polysomies were observed. Three (1.3\%) cases with Her-2/neu amplification coincided with a polysomy in the Top IIa analysis. Monosomies matched in $23(10.2 \%)$ cases. This comparison was highly significant $(\mathrm{p}<0,0005)$.
Her-2/neu and EGFR. In total, 221 (90.9\%) cases were compared using FISH analysis of Her-2/neu and EGFR. Twenty-two (9.1\%) cases could not be evaluated (see Table VIII). Three (1.4\%) cases of Her-2/neu amplification coincided with high polysomies in the EGFR analysis. The other cases with high polysomies of EGFR showed a nomal gene status (18 cases, $8.1 \%$ ), polysomies ( 2 cases, $0.9 \%$ ), and one monosomy $(0.5 \%)$ in the Her-2/neu analysis. Another seven cases of Her- $2 /$ neu amplification showed a low trisomy $(4 / 7,1.8 \%)$ and a low polysomy $(3 / 7,1.4 \%)$ in the EGFR evaluation. This analysis was significant $(\mathrm{p}=0.045)$. 
Top II a and EGFR. FISH analysis of Top IIa and EGFR was compared in 220 (90.5\%) cases. In total, 23 (9.5\%) cases were excluded from the evaluation (see Table IX). Four (1.8\%) cases revealed a polysomy in Top IIa analysis and a high polysomy in the EGFR analysis. Another four cases of Top IIa polysomy had a low polysomy in the EGFR evaluation. In 18 (8.2\%) cases a high polysomy was observed, but Top IIa gene status was normal. The only deletion $(0.5 \%)$ in the Top IIa analysis coincided with a high polysomy of EGFR. This comparison was significant $(\mathrm{p}<0.0005)$.

Survival analysis. All 243 patients were registered at the Munich Cancer registry. Complete follow-up data was available for 190 (78.2\%) patients, and 53 were lost to follow-up. By the end of data collection, only 52 (27.4\%) of the 190 women were still alive. Follow-up time ranged from 0-184 months. Survival time was divided into short ( $<3$ years), medium (3-7 years) and long ( $>7$ years). A total of $94(68 \%)$ of the 138 deceased patients showed a short, 37 (27\%) a medium, and $7(5 \%)$ a long survival time.

Survival and Her-2/neu. The Kaplan-Meier survival analysis for Her-2/neu was not statistically significant (IHC, $\mathrm{p}=0.71$; FISH, 0.143). Mortalities showed an almost equal percentage distribution. All patients with uncertain Her-2/neu status, $80.0 \%$ of the polysomy cases and $77.8 \%$ of the amplified cases succumbed to the disease. A long survival time could be observed for normal and amplified cases.

Survival and Top IIa. The Top IIa expression or chromosome status showed no association with survival in the Kaplan-Meier analysis (IHC, p=0.406; FISH, p=0.773). A total of $50.0 \%$ of the patients with a strong Top IIa expression succumbed to the disease. In cases of moderate or weak expression a long survival time could be observed.

A total of $75.0 \%$ amplified cases succumbed to the disease. Cases of monosomy ranked second with $72.7 \%$. The cases of polysomy and deletion showed a medium time of survival. Amplified cases and those with a normal Top IIa status reached a long survival time.

Survival and EGFR. No significant correlation between EGFR and survival could be observed in the Kaplan-Meier analysis (IHC, $\mathrm{p}=0.814$; FISH, $\mathrm{p}=0.702$ ). Almost $10 \%$ more patients with negative expression succumbed to the disease compared to those with a positive expression. Both groups showed a long survival time.

Mortality occurred most frequently in cases of low trisomy and low polysomy (both 63.8\%). High polysomy ranked second (61.9\%). A medium survival time could be observed in the group of high trisomy. The rest of the cases showed a long survival time.

\section{Discussion}

In our study population of FIGO stage III ovarian cancer patients, we analyzed aberrant gene copy or chromosome numbers by FISH of Her-2/neu, Top IIa and EGFR and their protein expression by IHC to evaluate a possible correlation with overall survival in these patients.
The majority of cases did not show an overexpression of the Her-2/neu oncogene or amplification in FISH analysis. Thus, the correlation of gene status and protein expression was highly significant. Survival analysis did not show a significant correlation with Her-2/neu receptor status, although the percentage of deceased patients was observed to increase from score 0 to 3 . For those patients with an overexpression (score 3 ) we found only short survival times in contrast to negative and uncertain cases that showed a long survival time.

Previous studies on Her-2/neu oncogene expression have shown vast differences with reported expression ranging from 1.8-76\%; however, some authors only differentiated between positive or negative expression and cases considered as $3+$ actually represented $2.3-22.2 \%$ (19-22). Our results in terms of survival are consistent with the majority of other published studies that did not find a correlation between Her-2/neu expression and survival, neither in ovarian cancer patients of all stages (23) nor in patients with advanced stage disease $(7,24)$. A meta-analysis on the prognostic value of Her-2/neu conducted by de Graeff et al revealed that overexpression is associated with poor overall survival (hazard ratio, 1.67), but the authors emphasize a considerable heterogeneity of the analyzed studies and that none of the studies reported a significant correlation between Her-2/neu expression and survival (20 studies with a total of 3055 patients published between 1990 and 2008 were analyzed) (11).

Coamplification of Her-2/neu and Top IIa existed in only six cases, suggesting that these genes are independent factors. High polysomies in the EGFR analysis also only coincided with three cases of Her-2/neu amplification.

Top IIa showed a high expression rate of $93.4 \%$, but IHC did not correlate with amplification in FISH analysis. Neither results from IHC nor from FISH analysis were able to predict survival, although patients with gene amplification of Top IIa showed the highest mortality rate. Only a few studies have investigated the impact of Top IIa on survival in ovarian cancer patients and the results remain inconsistent. Faggad et al (10) found an increased expression in patients with advanced stage disease, which is consistent with our findings of a high expression rate in FIGO stage III patients. However, they also report a correlation between high expression and short survival that cannot be confirmed in our study population. Others who also could not identify this correlation concluded that Top IIa expression is not useful as a marker for prognosis and may rather be an indicator for the effectiveness of anthracycline therapy $(9,16)$. This was shown for patients with breast cancer where Top IIa overexpression was able to predict responsiveness to anthracycline-based therapy $(25,26)$. Notably, as also shown in our cohort, gene amplification did not add predictive information.

Polysomy of Top IIa coincided with only four cases of high polysomy in the EGFR analysis. The data regarding EGFR expression and gene status are as inconsistent as those described for the previous two markers. EGFR expression ranges from $17-46.1 \%$ (27-29). Our data revealed a rather low expression of EGFR (13.1\%). FISH did not show any case of amplification and only two cases $(0.9 \%)$ showed a correlation between FISH and IHC (high polysomy and positive IHC). Survival analysis did not show a correlation between EGFR and outcome. 
In contrast to our findings, Lassus et al (27) found an amplification rate of $12 \%$ and a correlation of poor outcome, but a similar protein overexpression of $17 \%$. On the other hand, Tanaka et al (28) did not find any significant association between EGFR mutation and survival time. The lack of standardization in staining procedures and guidelines for interpretation of the EGFR assessment may be the major reason for the conflicting results across studies (18).

The challenge remains to identify prognostic markers in patients with advanced stage ovarian cancer that are able to differentiate between short- and long-term survivors and to find possible molecular targets to improve prognosis by targeted therapy. Of other tumor biological factors that have been studied previously, ErbB3 and Ki67 appear to predict a more favorable outcome or response to chemotherapy, but cannot be used as therapeutic targets yet $(8,9,30,31)$.

In conclusion, our study analyzed the effect of Her-2/neu, Top IIa and EGFR on survival for advanced stage ovarian cancer patients in a large study cohort. Our results underline the conflicting results that exist in the literature and emphasize the need for further studies in this subgroup of ovarian cancer patients.

\section{Acknowledgements}

We thank Helen Ryan for checking the spelling and writing of this manuscript, and Beate Luthardt and Andrea Sendelhofert for their excellent technical work.

\section{References}

1. Robert Koch-Institut und die Gesellschaft der epidemiologischen Krebsregister in Deutschland e. V. Berlin. Krebs in Deutschland 2003-2004 Häufigkeit und Trends. 6th edition, 2008.

2. Statistisches Bundesamt. Todesursachenstatistik in Deutschland. Fachserie 12, Reihe 4, pp1-114, 2008.

3. Deutsche Krebsgesellschaft e. V. Krebshäufigkeit - die aktuellen Zahlen, 2007.

4. Dietel M: Ovar: tumoren. In: Pathologie. Remmele W (ed). Vol. 4 2nd edition. Springer-Verlag, Berlin Heidelberg, pp92-123, 1992.

5. Olsen CM, Cnossen J, Green AC, et al: Comparison of symptoms and presentation of women with benign, low malignant potential and invasive ovarian tumors. Eur J Gynaecol Oncol 28: 376-380, 2007.

6. Cannistra SA: Cancer of the ovary. N Engl J Med 351: 2519-2529, 2004.

7. Goff BA, Muntz HG, Greer BE, et al: Oncogene expression: long-term compared with short-term survival in patients with advanced epithelial ovarian cancer. Obstet Gynecol 92: 88-93, 1998.

8. Kaern J, Aghmesheh M, Nesland JM, et al: Prognostic factors in ovarian carcinomas stage III patients. Can biomarkers improve the prediction of short- and long-term survivors? Int J Gynecol Cancer 15: 1014-1022, 2005.

9. Schindlbeck C, Hantschmann P, Zerzer M, et al: Prognostic impact of KI67, p53, human epithelial growth factor receptor 2 , topoisomerase IIalpha, epidermal growth factor receptor, and $\mathrm{nm} 23$ expression of ovarian carcinomas and disseminated tumor cells in the bone marrow. Int J Gynecol Cancer 17: 1047-1055, 2007.

10. Faggad A, Darb-Esfahani S, Wirtz R, et al: Topoisomerase IIalpha mRNA and protein expression in ovarian carcinoma: correlation with clinicopathological factors and prognosis. Mod Pathol 22: 579-588, 2009.

11. De Graeff P, Crijns AP, de Jong S, et al: Modest effect of p53, EGFR and Her-2/neu on prognosis in epithelial ovarian cancer: a meta-analysis. Br J cancer 101: 149-159, 2009.
12. Wang JC: Cellular roles of DNA topoisomerases: a molecular perspective. Nat Rev Mol Cell Biol 3: 430-440, 2002.

13. World Health Organization: Pathology \& Genetics. Tumours of the Breast and Female Genital Organs. IARC Press, Lyon, pp119, 2003.

14. Wolff AC, Hammond ME, Schwartz JN, et al: American Society of Clinical Oncology/College of American Pathologists guideline recommendations for human epidermal growth factor receptor 2 testing in breast cancer. Arch Pathol Lab Med 131: 18-43, 2007.

15. Remmele W and Stegner HE: Recommendation for uniform definition of an immunoreactive score (IRS) for immunohistochemical estrogen receptor detection (ER-ICA) in breast cancer tissue. Pathologe 8: 138-140, 1987 (In German).

16. Chekerov R, Klaman I, Zafrakas M, et al: Altered expression pattern of topoisomerase II $\alpha$ in ovarian tumor epithelial and stromal cells after platinum-based chemotherapy. Neoplasia 8: 38-45, 2006.

17. Pauletti G, Godolphin W, Press MF, et al: Detection and quantitation of HER-2/neu gene amplification in human breast cancer archival material using fluorescence in situ hybridization. Oncogene 13: 63-72, 1996.

18. Cappuzzo F, Hirsch FR, Rossi E, et al: Epidermal growth factor receptor gene and protein and gefitinib sensitivity in non-small-cell lung cancer. J Natl Cancer Inst 97: 643-655, 2005.

19. Serrano-Olvera A, Duenas-Gonzalez A, Gallardo-Rincón D, et al: Prognostic, predictive and therapeutic implications of HER2 in invasive epithelial ovarian cancer. Cancer Treat Rev 32: 180-190, 2006.

20. Lee ES, Lee Y, Suh D, et al: Detection of HER-2 and EGFR gene amplification using chromogenic in-situ hybridization technique in ovarian tumors. Appl Immunohistochem Mol Morphol 18: 69-74, 2010.

21. Vermeij J, Teugels E, Bourgain C, et al: Genomic activation of the EGFR and HER2-neu genes in a significant proportion of invasive epithelial ovarian cancers. BMC cancer 8: 3, 2008.

22. Dimova I, Raicheva S, Dimitrov R, et al: Coexistence of copy number increases of c-Myc, ZNF217, CCND1, ErbB1 and ErbB2 in ovarian cancers. Onkologie 32: 405-410, 2009.

23. De Graeff P, Crijns AP, Ten Hoor HK, et al: The ErbB signalling pathway: protein expression and prognostic value in epithelial ovarian cancer. Br J Cancer 99: 341-349, 2008.

24. Farley J, Fuchiuji S, Darcy KM, et al: Associations between ERBB2 amplification and progression-free survival and overall survival in advanced stage, suboptimally-resected epithelial ovarian cancers: a Gynecologic Oncology Group Study. Gynecol Oncol 113: 341-347, 2009.

25. Durbecq V, Paesmans M, Cardoso F, et al: Topoisomerase-II alpha expression as a predictive marker in a population of advanced breast cancer patients randomly treated either with single-agent doxorubicin or single-agent docetaxel. Mol Cancer Ther 3: 1207-1214, 2004.

26. Schindlbeck C, Mayr D, Olivier C, et al: Topoisomerase IIalpha expression rather than gene amplification predicts responsiveness of adjuvant anthracycline-based chemotherapy in women with primary breast cancer. J Cancer Res Clin Oncol 136: 1029-1037, 2010.

27. Lassus H, Sihto H, Leminen A, et al: Gene amplification, mutation, and protein expression of EGFR and mutations of ERBB2 in serous ovarian carcinoma. J Mol Med 84: 671-681, 2006.

28. Tanaka Y, Terai Y, Tanabe A, et al: Prognostic effect of epidermal growth factor receptor gene mutations and the aberrant phosphorylation of Akt and ERK in ovarian cancer. Cancer Biol Ther 11: 50-57, 2011.

29. Stadlmann S, Gueth U, Reiser U, et al: Epithelial growth factor receptor status in primary and recurrent ovarian cancer. Mod Pathol 19: 607-610, 2006.

30. Tanner B, Hasenclever D, Stern K, et al: ErbB-3 predicts survival in ovarian cancer. J Clin Oncol 24: 4317-4323, 2006.

31. Garcia-Velasco A, Mendiola C, Sanchez-Munoz A, et al: Prognostic value of hormonal receptors, p53, ki67 and Her2/neu expression in epithelial ovarian carcinoma. Clin Transl Oncol 10: 367-371, 2008 